\title{
Familia, origen social y capital cultural del alumnado de Trabajo Social
}

\author{
Luis Miguel Rondón García. Universidad de Málaga \\ Recepción: 16 de octubre de 2015 | Revisión: 16 de octubre de 2015 | Aceptación/Publicación: 19 de octubre de 2015 \\ Correspondencia: luirongar@uma.es \\ Citar: Rondon-Garcia, LM. (2015). Familia, origen social y capital cultural del alumnado de Trabajo Social. ReiDoCrea, 4, $334-342$.
}

Resumen: En este artículo se examinan los factores que influyen en la elección de los estudios, las perspectivas y el rendimiento académico de los estudiantes. También se analiza el impacto de estas circunstancias en la calidad de la educación en el difícil contexto actual, con los cambios sociales que se están produciendo por la crisis económica y social. Aunque los resultados se refieren a los estudiantes de pregrado, se pone de manifiesto cómo se reproduce clases de cultura tradicional y la segregación vertical de acuerdo a las expectativas sociales. En resumen, este estudio de investigación es analizar el potencial del capital cultural, origen social y familiar, en los estudiantes de Trabajo Social.

Palabras clave: Familia | Educación Superior

Family, Social Origin and Cultural Capital of Students in Social Work

Abstract: This article examines the factors that influence the choice of studies, prospects and academic performance of students. It also discusses the impact of these circumstances on the quality of education in the current difficult context, with the social changes that are occurring from the economic and social crisis. Although the results refer to undergraduates, it becomes clear how it reproduces traditional culture classes and vertical segregation according to social expectations. In short, this research study is to analyze the potential of cultural capital, social origin and family, in students of Social Work.

Keywords: Family | Higher Education

\section{Introducción}

Desde distintas prácticas discursivas se ha establecido la asociación entre el nivel socioeconómico de la familia, el capital social y cultural, y las expectativas de la familia, como elementos que condicionan la vida académica y social del alumnado universitario. Compartimos con Fumbuena (2011) que la familia como sistema de transmisión, es la mayor influencia en la estructura individual; influye de forma decisiva en el futuro comportamiento de clase, en los hábitos de estudio, en las expectativas sociales de los/as hijos/as, en la creación del mundo personal y social; cumple de una forma más determinante estas funciones que el resto de agentes de socialización. Aunque en los últimos años estamos viviendo una desinstitucionalización de las formas de familia tradicional, con el tránsito de las familias modelo a los diferentes modelos familiares, la familia sigue ocupando un lugar central en el ejercicio de estas atribuciones imbuidas por la sociedad (Rondón, 2012).

Como decía Rousseau, unos buenos padres valen por cien maestros. Por esta razón, para conocer las características que dan identidad al colectivo de Trabajo Social, resulta interesante conocer el capital familiar, el origen, como factores determinantes en la movilidad social y en la trayectoria académica o profesional. A partir de aquí podemos construir los argumentos que nos ayuden a comprender el perfil de los/as estudiantes de Trabajo Social. Estos procesos representan el vértice para el análisis de la futura mudanza social, que pueda derivarse en el origen de los y las estudiantes para la presente centuria. Nos permite aproximarnos a la evolución del colectivo en un futuro próximo, si relacionamos como influyen estas nuevas características en el rendimiento académico y en la situación social, así como en la elección racional de unos estudios o de unos perfiles sobre otros. 


\section{El capital cultural y social basado en la familia}

Las teorías del capital social y cultural han abierto una línea de investigación interesante para la sociología de las profesiones, de la educación y de las ciencias sociales en general, en cuanto al conocimiento de las causas que explican el éxito educativo y de rol (Herreros, 2002). La producción científica ha concluido en los diversos estudios, la importancia del capital social como variable independiente para explicar diversos fenómenos sociales, entre los cuales se encuentra el rendimiento académico, las expectativas sociales y la motivación para la elección del futuro profesional. Estos términos han sido utilizados en el estudio de las desigualdades sociales en el sistema educativo para explicar las ventajas culturales, sociales que los individuos y familias poseen, y que favorecen un nivel socioeconómico más elevado. Por lo tanto, las intersecciones de las condiciones económicas, sociales y culturales están presentes en la formación universitaria. Algunos segmentos de la clase social media alta y otras élites privilegiadas, motivan a sus descendientes para la elección estratégica de unos estudios y/o profesiones. Esta información social permite configurar un estatus conforme a su posición de clase, para que en la medida de lo posible sea un vehículo para la movilidad vertical y para la promoción social.

Sin embargo para Bourdieu (1997) el tiempo que dedican las familias dotadas de un fuerte capital cultural a sus hijos es mayor y dura casi todo el proceso de socialización. Es un capital porque se puede acumular a lo largo del tiempo. En cierta medida, la transmisión a los/as hijos/as, la asimilación de una generación a otra, es una condición para la reproducción social. Esto se hace plausible en los estudiantes mediante recursos, información y una adecuada objetivación de los fenómenos externos, conforme a sus reglas y claves. Para apropiarse de un bien cultural, es necesario ser portador del habitus cultural, de ahí la influencia de la familia de origen en el capital futuro de los hijos. El habitus de clase del individuo contribuye a su propia producción y reproducción, con componentes simbólicos. Esto implica que en estas clases sociales también existen unos hábitos, símbolos, modos y estímulos favorables al éxito académico y profesional. Al proceso socializador se añaden otros específicos, como una formación añadida en casa en cuanto a pautas culturales, comunicación, recursos, bienes culturales y tecnológicos, acceso a fuentes de información, apoyo extra.

En cuanto al capital social, Bourdieu (1989) lo define como los elementos constitutivos y los beneficios obtenidos por los individuos en su participación en redes sociales como son: los recursos procedentes de los miembros del grupo primario y los recursos propios que esta red genera. En sintonía con este autor, la familia tiene un papel fundamental en la construcción del capital social, por ser el agente de socialización por excelencia, según el cual los individuos interiorizan las normas de la cultura que les ha tocado vivir, transmite lo aprendido por la herencia cultural actual y pasada. Cada estatus trae consigo una serie de comportamientos y actitudes que se esperan de una persona según su historia familiar, social y cultural.

Por último, el capital económico lo componen la renta, los bienes y servicios a los que esta da acceso y la información social de partida. Cabe esperar que los alumnos/as procedentes de rentas altas, tengan más acceso a bienes y servicios educativos, y sobre todo accedan a estudios privados o titulaciones acordes a su posición de clase, así como a unos círculos sociales que facilitan el éxito social y opten por una serie de profesiones concretas. Este proceso se inicia en una etapa evolutiva anterior, en la educación secundaria, donde comienzan a elegirse itinerarios educativos humanísticos o técnico-científicos y comienza la formación adicional en idiomas, nuevas tecnologías, que muestran la senda para la construcción del futuro rol. En la misma línea argumental, no podemos olvidar la importancia de los grupos sociales hijos/as de otras 
familias con los que se relaciona el educando. Estos elementos, también representan un complemento añadido, por la identidad por adscripción que en estas interacciones se produce.

Según el análisis anterior, la evaluación de los resultados académicos va más allá de la simple verificación de la adquisición de competencias. Hay que explicar los resultados académicos y el comportamiento futuro de los titulados, en función el habitus cultural, el origen familiar y el capital del estudiante como sujeto del sistema educativo. Siguiendo con Bourdieu, en el homus academicus, encontramos las bases de análisis para aplicar esta teoría al campo de la Universidad, porque los distintos grupos académicos, se corresponden con los hábitos tradicionales entre ellos y con la división social del trabajo. La academia es una jerarquía social y cultural gobernada por el capital cultural que se deriva de la autoridad científica. La naturaleza del habitus cambia cuando se alteran las circunstancias humanas y la historia de un campo. No es casualidad que a pesar de la fuerte políticas de becas y de apoyo al estudio en décadas anteriores, encontremos diferencias de clase, género y capital, según el grado académico y la rama a la que pertenece la titulación, que implican una desigualdad social manifiesta. Es cierto que esto va cambiando, pero se necesita más tiempo para que se transformen estos hechos en cambios sociales. La igualdad de oportunidades no implica una igualdad de resultados, es decir, que los individuos terminen disfrutando de unas condiciones de vida semejantes. Aunque también es cierto que los pares que no han conseguido promoción y movilidad social, pueden proyectar a sus hijos para que sean ellos quienes continúen el camino iniciado por ellos, como prolongación psicológica y social de la familia.

\section{Materiales y métodos}

Esta investigación pretende conocer las expectativas y las características de la trayectoria social y familiar del alumnado de Trabajo Social, con las siguientes preguntas de investigación: ¿El origen de clase y el capital cultural tienen una idiosincrasia propia en el Trabajo Social? ¿Es cierto que es una carrera propia de clases sociales medias y de nivel socioeconómico medio-bajo? ¿Han cambiado estas circunstancias con el paso del tiempo?

Partimos de las siguientes hipótesis: El capital cultural del alumnado está asociado con las expectativas sociales y el rendimiento académico; el origen familiar y la clase social inciden en la elección de los estudios y en las posibilidades de movilidad social futura.

Los participantes fueron 280 estudiantes de segundo curso de Grado de Trabajo Social en las universidades de Málaga y Granada.

Atendiendo a los objetivos de investigación, la metodología ha sido básicamente cuantitativa. Hemos utilizado procedimiento de recogida de información, el cuestionario con preguntas cerradas de elección múltiple, con carácter individual y auto administrado. Se elaboró un cuestionario con doce ítems. Las variables de estudio se agrupan en los datos sociodemográficos, las expectativas sociales y académicas, el nivel socioeconómico, ocupación de los padres y estudios realizados por la familia.

Tras realizar el trabajo de campo, se procedió a la depuración y tabulación de los datos. Por el carácter descriptivo, exploratorio y explicativo del mismo, se han utilizado técnicas de procesamiento de la información a nivel descriptivo y otras inferenciales que han ayudado a interpretar las relaciones entre las variables señaladas. Al finalizar, con el objeto de sistematizar los resultados se ha representado los datos en la figura 1 , de forma gráfica, mostrando la intersección entre las variables. 


\section{Resultados y discusión}

En primer lugar y de forma breve, hacemos referencia a los resultados obtenidos en las variables sociodemográficas. Nos referimos a la estadística descriptiva, frecuencias y porcentajes. A continuación sistematizamos los principales datos obtenidos al respecto:

- El $78.2 \%$ son mujeres. Continúa la predominante de razón de mujeres sobre hombres. La presencia de la mujer a lo largo de la historia profesional del Trabajo Social como profesión es una contante. Este hecho está presente en su origen, desarrollo y, por el momento, futuro inmediato, tiene una estrecha relación con la evolución general de la profesión (Lorente, 2000). Los avances en materia de igualdad de género, parece que no modifican la construcción social del esquema anterior, es decir, la segregación vertical, la vinculación de las profesiones sociales o de ayuda a la mujer. Estos roles generalmente se reproducen por la familia, como mecanismo de transmisión, que hace que terminen experimentando trayectorias vitales distintas los hijos y las hijas.

- Un 73.9\% tienen entre 18 y 23 años. Es decir, la edad habitual en esta etapa académica. Esta información nos conduce a preguntarnos si las personas adultas o mayores de 45 años, que se están incorporando en mayor medida a la Universidad, están realizando estudios sociales o si por el contrario optan por carreras científicas o técnicas. Estos datos no son concluyentes en relación a la edad, pero estamos en condiciones de afirmar que cada vez más adultos eligen estos estudios para su promoción laboral y social.

- El modelo familiar predominante del alumnado es la familia nuclear conyugal en un $80.4 \%$. Un $13 \%$ lo componen las familias monoparentales y un $4.3 \%$ las reconstituidas. Debido a lo reciente de las nuevas formas de familia, todavía no se han visto reflejados los nuevos modelos familiares en esta generación. Atendiendo a los intentos cambios sociales, es predecible que los nuevos modelos familiares, cambien también el habitus y, el capital cultural de los futuros estudiantes.

El resto de variables específicas o segundo grupo, representan el tema central de este trabajo: el capital familiar y cultural de origen del alumnado. Siguiendo este planteamiento, en primer lugar, describimos los datos absolutos obtenidos del trabajo de campo en cada una de las variables que componen el capital cultural en las tablas 1, 2, 3 y 4. Posteriormente se cruzar las variables descritas para explicar las sinergias e interrelaciones que se producen, según los resultados obtenidos de la suma de todas ellas y que da lugar a un terminado nivel de capital cultural (figura 1). Comenzamos analizando los resultados de cada una de las variables que componen el capital cultural:

\begin{tabular}{|lc|}
\hline \multicolumn{2}{|c|}{ Tabla 1. Estudios de los padres } \\
\hline Estudios o nivel de instrucción & Porcentaje válido \\
\hline Sin estudios básicos finalizados & $21.74 \%$ \\
Estudios básicos & $28.26 \%$ \\
Estudios medios & $26-09 \%$ \\
Estudios universitarios & $13-04 \%$ \\
Doctorado o tercer ciclo & $5.52 \%$ \\
Otros & $4.35 \%$ \\
\hline
\end{tabular}

Como indica la tabla 1, se aprecia que casi la mitad de las familias de origen no tienen estudios, o si lo tienen solo han realizado los básicos. El porcentaje de universitarios y doctores es relativamente bajo (18.56\%). Esta variable indica un capital cultural bajo, un $50 \%$ proceden de unas familias con un nivel de instrucción medio-bajo. 


\begin{tabular}{|lc|}
\hline \multicolumn{2}{|c|}{ Tabla 2. Ocupación padre } \\
\hline Ocupación padre & Porcentaje válido \\
\hline Desempleo & $21.24 \%$ \\
Trabajo no cualificado & $52.04 \%$ \\
Trabajo cualificado o técnico & $5.52 \%$ \\
Titulado superior & $8.70 \%$ \\
Directivo o empresario & $5.3 \%$ \\
Otros & $8.4 \%$ \\
\hline
\end{tabular}

La mitad de los padres ocupan un empleo no cualificado (52.04\%), con un porcentaje muy bajo de titulados superiores y/o directivos (14\%). Esto refuerza nuestra hipótesis inicial de un capital medio- bajo, aunque ligeramente superior a la variable anterior.

\begin{tabular}{|lc|}
\hline \multicolumn{2}{|c|}{ Tabla 3. Ocupación madre } \\
\hline Ocupación madre & Porcentaje válido \\
\hline Desempleo & $34.28 \%$ \\
Trabajo no cualificado & $30.49 \%$ \\
Trabajo cualificado o técnico & $17.38 \%$ \\
Titulada universitaria o superior & $13.4 \%$ \\
Directiva o empresaria & 4.35 \\
Otras & $1 \%$ \\
\hline
\end{tabular}

En el caso de las madres, los datos son parecidos en términos absolutos, aunque ligeramente superiores a los obtenidos con los padres. Hay que tener en cuenta, que el porcentaje de desempleo es mayor en las madres, de ahí, que no sea significativa la diferencia estadísticamente hablando. El $30.49 \%$ tiene un empleo no cualificado. En cambio, el trabajo cualificado o superior es mayor que el de los padres $(30.78 \%)$. Existe un cierto consenso en los discursos en torno a la identidad profesional del colectivo de Trabajo Social, que considera la variable género como eje transversal y vértice para la explicación de la división social del trabajo. En este caso observamos que las madres cualificadas y universitarias, motivan hacia carreras sociales a sus hijos e hijas.

\begin{tabular}{|lc|}
\hline \multicolumn{2}{|c|}{ Tabla 4. Nivel socioeconómico } \\
\hline Renta media anual & Porcentaje válido \\
\hline Menos de $15.000 €$ & $30.43 \%$ \\
Entre 15.001 y $20.000 €$ & $26.09 \%$ \\
Entre 21.000 y $26.000 €$ & $23.91 \%$ \\
Entre 27.000 y $32.000 €$ & $8.70 \%$ \\
Entre 33.000 y $38.000 €$ & $8.70 €$ \\
Entre 39.000 y $40.000 €$ & $2.12 €$ \\
Más de $45.000 €$ & $1 \%$ \\
\hline
\end{tabular}

La tabla número 4 muestra que la mayoría (80.43\%) tiene una renta media inferior a los $26.000 €$ anuales. Un $30.43 \%$ de las familias de origen se encuentra en situación de pobreza relativa. El nivel de rentas es medio-bajo, lo cual condiciona también la posición social y de clase. El elevado número de desempleados en el momento actual puede influir en los datos y es un indicador a tener en cuenta para explicar la renta baja.

De acuerdo con los datos obtenidos en función del capital familiar, social y económico de los discentes de Trabajo Social, se distinguen dos grandes grupos. En primer lugar, los que provienen de clase media alta, con mayores recursos y mecanismos para la inserción social, que en este caso y en función de la renta representan el $20 \%$ del total. En segundo lugar, los que provienen de clases medias y media bajas (un $80 \%$ del total) en situación de vulnerabilidad social. En este último caso se confirma que 
optan por el estudio de carreras sociales, que posibilita una movilidad de clase más suave que otras opciones.

Para completar los resultados del capital cultural en torno al colectivo y tomando como referencia el análisis por conglomerados, realizamos una clasificación de los resultados cuando los objetos de un mismo grupo son similares entre sí o estadísticamente significativos. Utilizamos el análisis de clúster, a partir del estudio de Figueras (2001). Es una técnica estadística multivariable, cuya finalidad es dividir un conjunto de objetos en grupos, de forma que los perfiles de los objetos en un mismo grupo sean muy parecidos entre sí. Por esta razón, clasificamos a los estudiantes en tres grupos: los que proceden de clases media bajas; los que provienen de clases medias; los de clase media alta. A continuación, explicamos los criterios de elección de cada uno de estos tres grupos establecidos.

\begin{tabular}{|c|c|}
\hline \multicolumn{2}{|r|}{ Tabla $n^{0} 5$. Grupos de capital familiar y social } \\
\hline Capital & Definición o características \\
\hline Muy bajo & $\begin{array}{l}\text { Se representa por estudiantes que combinan a padres y madres sin estudios, un nivel de } \\
\text { renta bajo (menos de } 15.000 € \text { anuales), y una profesión de los ascendientes no } \\
\text { cualificada. }\end{array}$ \\
\hline Normal bajo & $\begin{array}{l}\text { Se trata de estudiantes con padres y madres con estudios básicos o medios finalizados, } \\
\text { con un índice socioeconómico medio- bajo (entre } 15.000 \text { y } 26.000 € \text { anuales) y una } \\
\text { profesión de los padres cualificada, pero no universitaria. }\end{array}$ \\
\hline Medio alto & $\begin{array}{l}\text { Se determina por padres y madres con estudios universitarios o cargos directivos, con un } \\
\text { nivel de renta medio- alto (más de } 27.000 € \text { anuales) y una profesión de los padres } \\
\text { universitaria o análoga. }\end{array}$ \\
\hline
\end{tabular}

Conforme a los tres grupos establecidos en la tabla anterior, a continuación en la figura 1 se representan los resultados finales obtenidos en las cuatro variables estudiadas (renta media, estudios familia, ocupación del padre y ocupación de la madre). En la figura podemos visualizar el capital cultural y familiar según los tres niveles o grupos establecidos en la tabla número 5 . La fórmula del capital cultural, a partir de las teorías de Bourdieu, es el resultado de la suma de los estudios de los padres + la ocupación de los padres + el nivel socioeconómico de la familia (Casilla, Chain y Jácome, 2007). Según lo expuesto, el sumatorio final capital cultural y familiar del alumnado es el siguiente:

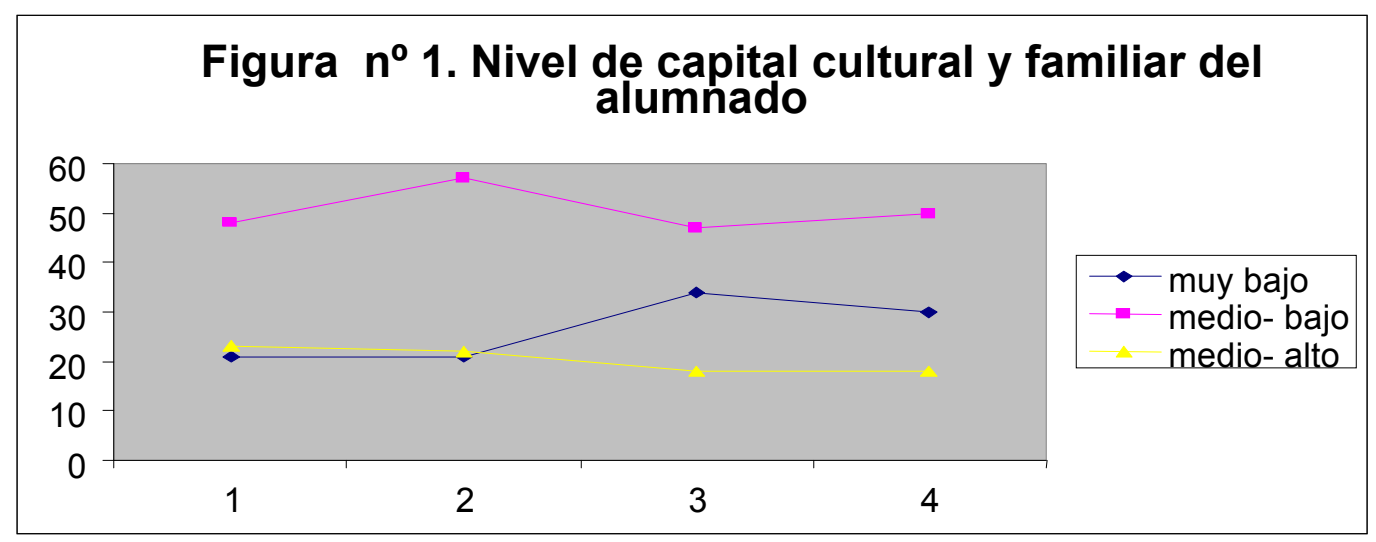

Podemos apreciar como el grupo mayoritariamente dispone de un capital cultural medio-bajo, siendo más acusada esta situación en el caso del nivel socioeconómico. En cambio el capital medio alto, representa una parte de la población pequeña, aunque con homogeneidad en las cuatro variables, es decir, una renta medio alta, nivel de estudios medio-altos y unas ocupaciones de los padres con un estatus acorde a su posición de clase. El sector de capital cultural muy bajo, es poco significativo 
estadísticamente hablando. Concluimos que el nivel socioeconómico y el origen familiar de los/as estudiantes en Trabajo Social es medio-bajo y en consecuencia el realizar la titulación universitaria, puede suponer una oportunidad para la movilidad social, que no tienen por regla general sus ascendientes.

Por otra parte, entendemos que estas variables también han influido en el rendimiento académico anterior durante el bachillerato, teniendo en cuenta, que la nota de corte para el acceso a estos estudios ronda en ambas universidades en torno a los 5 puntos. En cambio, contamos con la alta motivación del alumnado como factor añadido al capital, porque la mayoría de ellos han elegido la carrera como primera opción.

\section{Conclusiones}

Según lo expuesto en las consideraciones anteriores, el capital cultural es el resultado de la intersección del capital familiar y el capital social. Por eso, podemos identificar tipos diferentes de alumnado en cada titulación y/o profesión, de acuerdo con las disposiciones culturales y familiares aprendidas a lo largo de todo el proceso de socialización familiar.

Con base a los resultados obtenidos, hemos querido poner en evidencia la existencia de una diversidad de tipos de estudiantes en función de su capital cultural y familiar. Primero, para romper con las falsas representaciones sociales que los consideran a todos iguales. Pero también para poner sobre la mesa, el ideario de la conquista de la igualdad de oportunidades, porque aunque el alumnado tenga a priori una igualdad de condiciones, las reglas, el origen y su situación social, se puede entender en términos desiguales.

A lo largo de todo el proceso, hemos explorado el potencial explicativo del capital cultural, social, en la elección del Grado en Trabajo Social, para conocer el perfil social y los condicionantes en la elección de esta carrera, que inciden también en el rendimiento académico. Consideramos que los aspirantes a la educación superior están desigualmente dotados, desde el primer momento que acceden a la misma, al poseer diversos volúmenes de capital cultural, con unas circunstancias desiguales y de entrada a los procesos de selección que favorecen un determinado tipo de perfil de estudiantes. A pesar del principio de igualdad de oportunidades, implantado en nuestra sociedad tras más de tres décadas de democracia, parece que en este caso específico no implica una igualdad de resultados, en el sentido de que existen profesiones 0 titulaciones segregadas verticalmente, que son más frecuentes en ciertos grupos sociales y con connotaciones de género.

Según los términos que hemos analizado sobre los componentes del capital cultural de nuestros estudiantes, las determinaciones sociales y familiares tienen un valor específico y funcionan como un factor de diferenciación para garantizar el ingreso a la universidad en unos perfiles o titulaciones concretas y, para asegurar una determinada trayectoria profesional. Esta desigual distribución del capital, está significativamente asociada con las desigualdades familiares. A menor capital cultural, se espera también menos rendimiento académico y/o posibilidades de promoción social. Por estas razones, el rendimiento en la institución universitaria debe explicarse no solo conforme a aptitudes intelectuales, académicas o individuales, sino como un conjunto de factores globales o externos que son determinantes (entorno social, cultural y familiar).

La influencia de la historia profesional y laboral de los padres en la canalización o realización de expectativas es un factor explicativo en la elección de los estudios de 
Trabajo Social. Podemos establecer que los estudiantes y sus familias, construyen las estrategias apropiadas para la selección de la carrera a la que aspiran como parte de una estrategia social. Los resultados nos muestran la existencia de una fuerte segmentación social del sistema universitario español, que debe superar, para lograr una mayor equidad social, educativa y de clase. El cambio social y el grado de desarrollo de estas cuestiones en los últimos tiempos, no ha tenido un paralelismo en la movilidad social, en las posibilidades de los grupos y clase sociales medias o mediabajas. Se hace notoria la desigualdad y el riesgo de los más frágiles para lograr la promoción social.

Según Bourdieu (1988) "detrás de las relaciones estadísticas entre el capital educativo o el origen social, se ocultan relaciones entre grupos que mantienen a su vez relaciones diferentes, a veces antagónicas, con la cultura, según las condiciones en las que han adquirido su capital cultural y los mercados en los que pueden obtener de él un mayor provecho". Según esto, las familias con capital cultural alto, sirven de modelo de comportamiento para los hijos e hijas. Facilitan la ayuda necesaria para realizar un comportamiento determinando, sirven de modelo de aprendizaje para estrategias futuras. En este aspecto es donde encontramos las mayores carencias en clases medias y bajas, es decir, en el perfil de nuestro alumnado.

Los planteamientos hasta aquí expuestos cuestionan el corolario de la idealización del sistema de clases en función del mérito y la capacidad. El sistema educativo produce como efecto la legitimación de la estructura de clases, aportando una específica contribución a que esa estructura se reproduzca (Del Campo, 1988). Es importante recordar que las expectativas que tienen los grupos privilegiados con su descendencia, es transmitido a las siguientes generaciones, y funciona como plus añadido de motivación para conquistar el éxito social. Con la actual crisis económica y de las profesiones sociales, la situación que atraviesan las clases medias es dura, con un claro empeoramiento de las condiciones de vida, mayor estrés y menos tiempo para dedicar a los hijos/as. Esto puede verse reflejado en la idea, valores que transmiten a los mismos y en una clara falta de expectativas sociales, tanto de las familias como del propio alumnado en la elección para el futuro o desarrollo profesional. Por otra parte, la socialización y expectativas que las familias depositan y reproducen en las hijas, no es la misma que los hijos, porque la igualdad y el género, son principios, que aún no están del todo instaurados en la vida familiar y social. Esto condiciona también el tipo de estudios que eligen las alumnas, dentro del imaginario social vinculando a la mujer de profesiones de ayuda, dedicadas al cuidado y sociales.

Por último comentamos otros factores externos que pueden condicionar la elección de los estudios. Conjuntamente a estas variables culturales, familiares y sociales, hay carreras, como ocurre con las ciencias sociales en general, y en particular en el Trabajo Social, que tienen un imaginario colectivo, conformado a lo largo de una tradición histórica y cultural. Factores que hemos constatado en el estudio que tienen un nexo común en el Trabajo Social, al tratarse de una carrera de perfil socioeconómico medio, que proporciona unas supuestas garantías de éxito y estatus en clases media-bajas, con una movilidad social moderada. Esto contribuye a sostener en hijos/as con un capital medio y bajo, elecciones y vocaciones, relacionadas directamente con su perfil económico y sus perspectivas de futuro. También existe una vertiente complementaria que influye en el deseo, basada en la realización personal, y las posibilidades de inserción socio-laboral en el mercado de trabajo. En este caso la situación de las políticas de bienestar y del mercado, son factores que van a condicionar aún más el acceso a la Universidad y a unas titulaciones frente a otras. 
A tenor de lo expuesto, consideramos necesario fomentar la investigación y producción social en torno a los perfiles del alumnado, su capital familiar y social, para comprender los resultados académicos, la motivación y sus expectativas; así poder evaluar el grado de impacto de los cambios sociales. Por lo tanto, se pretende conocer desde una perspectiva holística, todos los componentes que explican los resultados del proceso formativo, más allá de las competencias, analizando el impacto de la crisis económica en la motivación, rendimiento académico y expectativas de futuro en el alumnado.

\section{Referencias}

Bourdieu, P. (1988). La distinción. Criterios y bases sociales del gusto. Madrid: Taururs.

Bourdieu, P. (1989). Outline of a theory of practice. Cambridge: University Press.

Bourdieu, P. (1991). El sentido práctico. Madrid: Taurus.

Bourdieu, P. (1997). Razones prácticas. Sobre la teoría de la acción. Barcelona: Anagrama.

Bourdieu, P. (1997). Capital cultural, escuela y espacio social. México: Siglo XXI.

Casillas M, Chain, P y Jácome, N (2007) Origen social e los estudiantes y trayectorias estudiantiles en la Universidad veracruzana. Revista de Educación Superior, 142(2), 7-29.

Del Campo, S. (1988). Tratado de Sociología. Volumen 2. Madrid: Taurus.

Figueras, M. (2001). “Análisis de conglomerados o clúster”. http://www.5campus.org/lección/cluster

Fombuena Valero. (2011). Un estudio de las familias de origen de los y las trabajadoras sociales desde el modelo contextual. Comunitania, International Journal of Social Work and Social Sciences, 2, 23-37.

Garvía, R. (1998) Conceptos fundamentales de Sociología. Madrid: Alianza editorial.

Herreros, F. (2002). ¿Son las relaciones sociales una fuente de recursos? Una definición de capital social?. Papers 67, $129-148$.

Lorente Molina, B. (2000). Género, profesión y cultura. Una aproximación al estudio de la identidad de los trabajadores sociales. Revista de Política Social y Servicios Sociales, 49, 97-114.

Lorente Molina, B. (2004). Género, ciencia y trabajo. Las profesiones feminizadas y las prácticas de cuidado y ayuda social. Scripta ethnologica, 26, 39-53.

Rondón García, LM. (2012). Bases para la Mediación Familiar. Valencia: Tirant lo Blanch. 